\title{
Environnement et Spermatogenèse
}

\author{
L. BUJAN \\ CECOS Midi-Pyrénées - Centre de Stérilité Masculine \\ Laboratoire sur la Fertilité Humaine (Université Paul Sabatier) \\ CHU La Grave Place Lange 31052 Toulouse cedex France
}

\section{RÉSUMÉ}

Plusieurs études récentes rapportent une altération de l'appareil reproducteur chez le mâle ainsi que des différences régionales suggérant un lien entre environnement et spermatogenèse. Les histoires du dibromochloropopane, du distilbène, du gossypol, démontrent l'influence d'une exposition professionnelle, médicamenteuse ou d'un mode de vie sur l'appareil reproducteur. Dans cette revue sont envisagés les facteurs chimiques (pesticides, métaux, solvants ...) physiques (radiations ionisantes, non ionisantes, chaleur), et les éléments du mode de vie (chaleur, position, habillement, alcool, tabac, stress, sport, alimentation) pouvant avoir une action délétère sur la spermatogenèse. L'hypothèse des perturbateurs endocriniens est discutée à la lumière d'études récentes faisant état de différences régionales. Les liens entre environnement et spermatogenèse posent la question du type d'études à mener et celle des outils à utiliser. Si des études pluridisciplinaires sont encore nécessaires afin de précisément déterminer le rôle de ces facteurs dans la décroissance séculaire et les variations régionales de la spermatogenèse, il est suggéré que le sperme puisse être un indicateur de santé publique et que le principe de précaution soit institué et se traduise concrètement : 1) par une réelle action de prévention en milieu professionnel, 2) par l'évitement d'un facteur de risque découvert lors des consultations pour infécondité, 3) par la non-utilisation de substances n'ayant pas fait la preuve de leur innocuité dans les études toxicologiques chez l'animal. Par ailleurs, la mise en place d'une spermovigilance pourrait être souhaitable.
Mots clés : environnement, infertilité, sperme, déclin, facteur de risque, stérilité, toxiques, mode de vie, profession, revue

Des études récentes ont fait état de l'augmentation de l'incidence d'anomalies de l'appareil de reproduction masculin dans l'espèce humaine. Ainsi une augmentation de la fréquence du cancer du testicule [1,37], de la cryptorchidie [18], de l'hypospadias [121] et peut-être du cancer du sein [29] ont été rapportés. Si quelques études avaient, il y a plus de dix ans, émis l'hypothèse d'une baisse du nombre de spermatozoïdes produit par l'homme dans les 50 années précédentes [86, 72, 8], d'autres avaient contredits cette hypothèse [76]. En 1992 une meta-analyse portant sur 61 études incluant au total 14947 hommes normaux, relance le débat en montrant une baisse du nombre de spermatozoïdes (de 133 à 66 x $106 / \mathrm{ml})$ et du volume du sperme $(3,40$ à 2,75 $\mathrm{ml}$ ) entre 1938 et 1990 [19]. Les résultats de cette étude ont été largement discutés dans la littérature [91, 31, 70] et plusieurs équipes dont la notre ont analysé leurs données avec des résultats allant dans le sens de la baisse pour les uns tandis que les autres ne trouvaient pas de changement. Ces différences peuvent s'expliquer par les méthodologies d'analyse employées, les effectifs étudiés, le type de sujet inclus dans les études (témoins, donneurs, hommes inféconds,..), la durée de l'étude ou les différences géographiques. Ainsi 
il est intéressant de constater qu'en Europe la région qui a le nombre de spermatozoïdes le plus élevé est la Finlande, ce pays ayant également la prévalence la plus basse en ce qui concerne le cancer du testicule. Dans notre pays l'étude de Auger et al. [5] ont mis en évidence une diminution de 2,1\% par an de la concentration de spermatozoïdes entre 1973 et 1992 dans une population homogène de 1351 donneurs et ceci en s'entourant de nombreuses précautions méthodologiques. De plus cette étude montre une diminution parallèle de la qualité (\% de mobilité, \% de formes normales] des spermatozoïdes. Dans notre équipe dans une étude, certes à effectif plus modeste, nous n'avons pas mis en évidence de baisse du nombre de spermatozoïdes entre 1977 et 1992 [12] mais, fait surprenant, notre concentration en 1977 semble plus basse que celle de la région parisienne au début de l'étude. En dehors des biais liés aux techniques d'analyses qui devront être éliminés dans les études prospectives prochaines ceci fait apparaître des différences régionales confortées dans une étude récente de la Fédération Française des CECOS [32]. Parallèlement à ces atteintes décrites concernant l'espèce humaine, des atteintes de la spermatogenèse, de l'appareil reproducteur ou une féminisation de certains caractères ont été rapportées dans de nombreuses espèces animales comme les alligators du Lac Apopka en Floride [49, 48], certains oiseaux [38, 39], des poissons [85, 2], des tortues [6], des panthères [30]. Les atteintes de l'appareil reproducteur mâle tant chez l'homme que chez l'animal ainsi que les différences régionales constatées posent le problème d'un lien entre environnement et spermatogénèse / appareil reproducteur mâle. Cette revue a pour objectif de décrire les liens entre spermatogenèse et environnement en prenant l'environnement dans son sens le plus large incluant des facteurs chimiques, physiques aussi bien que le mode de vie des individus.

\section{ENVIRONNEMENT ET SPERMATOGE- NESE : DES EXEMPLES HISTORIQUES}

\section{Le Dibromochloropropane (DBCP) est} un exemple type associant une exposition professionnelle et l'atteinte de la spermatogenèse. Le DBCP est un pesticide qui a été largement utilisé et dont l'action délétère sur la spermatogenèse $d u$ rat avait été mise en évidence dès 1960 [115]. Ce n'est que 17 ans après soit en 1977 que Whorton et al [124] publient une étude réalisée à la demande d'ouvriers d'une usine de production qui avaient des difficultés à avoir des enfants. Sur 25 ouvriers 9 présentaient une azoospermie et 3 une oligospermie avec pour les azoospermes une augmentation de la FSH. Plusieurs études ont ensuite été menées chez des travailleurs exposés [revue : 123] avec la mise en évidence d'un effet dose lié à la durée d'exposition, d'une récupération possible inversement corrélée à la durée d'exposition et peu évidente chez les sujets azoospermes. Par ailleurs dans certaines études, ont été rapportées, chez les personnes exposées, une réduction de la fertilité $[124,68,96]$, une augmentation du risque d'avoir une fausse couche spontanée ( $R R \times 3$ ) [59] et une diminution du sex ratio [96]. Ces études ont abouti à l'interdiction du DBCP dans les pays développés dès 1979. Il est toutefois intéressant de savoir que les compagnies chimiques qui fabriquaient le DBCP continuaient son exportation vers les pays en voie de développement comme par exemple le Costa Rica où récemment [1984] des employés agricoles rendus stériles par le DBCP ont été indemnisés 37200 francs à condition que ni eux, ni leur famille, ni leur éventuels descendants ne se retournent contre les compagnies.

2. L'utilisation du Distilbène (Diéthylstilbestrol DES) est un exemple de l'utilisation pharmacologique d'une molécule avec des conséquences sur l'appareil reproducteur féminin et masculin. Le DES a été synthétisé en 1938. Cet œstrogène synthétique fut donné à des millions de femmes enceintes dans le but de prévenir les fausses couches et les complications de la grossesse et ce jusqu'aux années 1970, malgré la publication de Dieckmann et al. [24] qui, dès 1953, montrait, lors d'un essai randomisé en double aveugle sur 1646 patientes, l'inefficacité du distilbène dans ces indications. Si les conséquences de l'administration à la mère de DES sont bien connues chez les filles en raison de l'apparition d'un cancer particulièrement rare chez certaines patientes [52] il n'en a pas été de même chez les enfants mâles, la pathologie induite étant 
moins bruyante. Toutefois plusieurs études font état chez les mâles exposés au DES in utero d'une augmentation de la fréquence de sténose du méat urinaire, de l'hypospadias, de kystes épididymaires, de testicules hypotrophiques, de cryptorchidies comparée à la fréquence dans les groupes contrôles [51, 45, 44, 109, 122].

Concernant la spermatogénèse, plusieurs études rapportent une altération $[44,106,109$, 122] alors que Andonian et Kessler ne montrent pas de différence mais sur un effectif réduit de 24 exposés et 24 non exposés [3]. Une étude récente a repris la cohorte de Dieckmann et a analysé 253 hommes ayant été exposés au DES in utero et 241 ne l'ayant point été [125]. Le taux d'anomalies de l'appareil génital est significativement augmenté chez les mâles exposés (15\% VS 5\% : multiplié par 3] et l'homme exposé in utero avant la $11^{\text {ème }}$ semaine de grossesse a deux fois plus d'anomalies que ceux qui ont été exposés après.

La production de sperme ne fut pas étudiée ici mais aucune différence ne fut mise en évidence entre les deux groupes en ce qui concerne les paramètres de la fertilité (nombre d'enfants, temps nécessaire pour la conception...).

Lincidence du cancer de testicule chez les hommes exposé in utero au DES pourrait être double ( $\mathrm{RR}=2,1 \quad[1,3-3,3])$ [méta analyse 114] que dans la population générale mais ceci reste à confirmer et il serait particulièrement intéressant que l'étude de la fréquence du cancer du testicule soit menée sur la cohorte de Dieckmann.

Enfin rappelons que les souris mâles exposés in utero au DES présentent une fréquence augmentée de kystes épididymaires, d'hypotrophie testiculaire, de cryptorchidies, d'altération de la production de sperme et de la fertilité, des atypies cellulaires de la prostate [79, 88] ainsi qu'une hyperplasie des cellules de Sertoli, des tumeurs interstitielles du testicule, une métaplasie des vésicules séminales et un adénome du rete testis [87]. Ces anomalies rencontrées chez la souris, identiques aux anomalies décrites chez l'homme indiquent que les résuitats des modèles animaux peuvent être parfois informatifs et importants pour l'espèce humaine.
3. Enfin, un dernier exemple qu'il est bon de rappeler brièvement, l'histoire de la découverte du Gossypol qui a été par la suite utilisé comme produit contraceptif masculin chez des milliers d'hommes avec une réelle efficacité mais également des effets secondaires et surtout un pourcentage d'irréversibilité important ayant fait arrêter son usage [97]. Cet exemple illustre parfaitement l'influence d'un mode de vie sur la spermatogenèse. En fait, ce produit est extrait de l'huile des graines de coton et sa découverte est due au fait que dans une région chinoise, les femmes cuisinaient avec de l'huile de coton, ce qui avait pour conséquence de rendre stériles les hommes de cette région ou les hommes qui venaient $\mathrm{y}$ habiter alors que certains d'entre eux partant vivre ailleurs pouvaient par la suite avoir des enfants.

Ainsi ces trois exemples montrent qu'une altération de l'appareil reproducteur masculin peut survenir à la suite d'une exposition professionnelle, d'une exposition médicale ou d'une exposition à un facteur de risque résultant du mode vie.

\section{FACTEURS CHIMIQUES}

Si de nombreux produits étaient connus pour avoir une action délétère sur la spermatogenèse chez l'animal ou l'homme, les atteintes décrites tant dans la faune sauvage en relation avec l'exposition à des pesticides, que dans l'espèce humaine concernant l'appareil reproducteur ont fait émerger la notion de perturbateurs endocriniens [107].

On peut définir comme perturbateur ou modificateur endocrinien un agent exogène qui interfère avec la synthèse, le stockage, le transport, le métabolisme, la fixation, l'action ou l'élimination des hormones naturelles responsables de l'homéostasie et de la régulation des mécanismes du développement [21]. Ces agents exogènes vont donc venir perturber le développement de l'individu lors d'une exposition in utero ou le fonctionnement normal de l'individu lors d'une exposition chez l'adulte. Ainsi le DDT puissant insecticide interdit dans les pays développés mais encore exporté dans d'autres pays est une molécule répondant à la définition des perturbateurs endocriniens. La 
plupart de ces métabolites possèdent une action de type oestrogénique et dès 1950 la féminisation de jeunes coqs à qui on avait administré du DDT était rapportée [16]. Très récemment en plus de l'action estrogénique du DDT, a été mise en évidence une action antiandrogénique d'un des principaux métabolites du DDT le pp'-DDE, tant in vitro qu'in vivo [58]. Cet exemple est particulièrement intéressant car il montre la complexité des perturbateurs endocriniens (œstrogène like et antiandrogène pour le DDT) largement répandus dans l'environnement et parfois à demi vies très longues. De très nombreuses substances utilisées dans l'agriculture, l'industrie ou la vie quotidienne semblent être des perturbateurs endocriniens (polychlorobiphényls : 209 substances, dioxines : 75, furanes : 135,...) [20] ainsi que les produits végétaux comme des phytoestrogènes. Deux études récentes soulignent les variations régionales concernant les perturbateurs endocriniens.

Schmid et al. [105] s'intéressant aux organochlorés, produits largement utilisés dont l'absorption peut se faire par inhalation, par voie cutanée ou par l'alimentation, après contamination de la chaîne alimentaire, vont étudier les différences régionales de concentration plasmatiques d'un métabolite du DDT, le DDE et de polychlorobiphényls. Le groupe témoin étant constitué de sujets allemands, ils vont étudier quatre groupes de réfugiés ayant quitté leur pays depuis 8 jours en moyenne (de 3 à 20 jours) : yougoslaves, ancienne U.R.S.S., asiatiques, africains. Les concentrations plasmatiques de DDE sont élevées chez les asiatiques, les réfugiés d'U.R.S.S. et les africains, reflétant ainsi l'usage intensif du DDT dans ces régions. Ils ne retrouvent pas de lien entre la profession et la concentration plasmatique en DDE émettant l'hypothèse d'une contamination générale notamment par la chaîne alimentaire. Par ailleurs, il est intéressant de noter que bien que l'usage du DDT ait été interdit en Allemagne dès 1976, son métabolite est encore dosable, 20 ans après, dans le plasma des hommes allemands. Concernant les polychlorobiphényls, la concentration la plus importante est retrouvée dans le groupe allemand et dans une moindre mesure dans le groupe issu de l'U.R.S.S. Là aussi, pas de lien entre la profession et la concentration plasmatique reflétant une contamination plus générale à mettre en rapport avec l'industrialisation du pays.

L'étude de Garcia-Rodriguez et al. [41] montre un lien entre l'utilisation de pesticides et le taux d'orchidopexie pour cryptorchidie. Ces auteurs ont étudiés la fréquence de l'orchidopexie pour 10.000 habitants et la quantité de pesticides employés dans la province de Granada, dans le sud de l'Espagne. Il semble apparaître un lien entre ces deux paramètres, le taux d'orchidopexie étant maximum dans la zone ou l'on utilise le plus de pesticides dans une agriculture où ces produits sont utilisés dans des serres.

Ces deux études récentes illustrent bien les liens entre exposition à un facteur de risque et environnement au sens large (utilisation professionnelle, habitude de vie, chaîne alimentaire ...). De très nombreux produits sont connus pour avoir une action délétère sur la spermatogénèse si ce n'est chez l'homme, du moins chez l'animal (tableau 1). Ainsi, des pesticides comme le DBCP, l'éthylène dibromide (EDB), le carbaryl (Sevin), le chlordécone (Kepone) ont des effets délétères connus chez l'animal. Chez des ouvriers utilisant l'EDB en fumigation sur des plantations de papaye, il a été rapporté un effet négatif sur la quantité et la qualité de la production de sperme [99] ainsi qu'une atteinte de la fertilité dans une usine de production [126]. Les études concernant le carbaryl, largement utilisé dans de nombreux insecticides ont simplement montré une augmentation des spermatozoïdes morphologiquement anormaux [127]. Une oligospermie et une asthénospermie ont été rapportées chez des hommes chroniquement exposés au chlordécone [50] et ayant par ailleurs des effets neurologiques de ce pesticide. Les études concernant l'influence des pesticides chez l'homme se heurtent d'une part, au fait qu'en dehors de populations très particulières, il s'agit le plus souvent d'une exposition à un nombre important de principes actifs utilisés durant l'année avec des variations saisonnières dans leur utilisation et d'autre part, une difficulté à mesurer les concentrations absorbées, du fait des méthodes de travail et de protection. Lors d'une étude 
Tableau 1 : Agents chimiques professionnels : action délétère chez l'animal ou l'homme (+: effet retrouvé dans au moins une étude. -: absence d'action dans les études effectuées. ?: inconnu) [ref. : 14, 110].

\begin{tabular}{|c|c|c|}
\hline & Effet & tère chez \\
\hline & Animal & Homme \\
\hline $\mathrm{DBCP}$ & + & + \\
\hline Ethylène dibromide & + & + \\
\hline Carbaryl & + & +- \\
\hline Chlordecone & + & + \\
\hline Epichlorhydrine & + & - \\
\hline $\begin{array}{l}\text { Dioxines et dérives } \\
\text { agent orange }\end{array}$ & + & +- \\
\hline Polychlorobiphenyls & + & - \\
\hline Cadmium & + & $?$ \\
\hline Manganèse & + & + \\
\hline Plomb & + & + \\
\hline Mercure & + & $+?$ \\
\hline Bore & + & $?$ \\
\hline Acétone & + & $?$ \\
\hline Diaminotoluène & + & - \\
\hline Carbon disulfure & + & + \\
\hline Dibromoethane & + & $?$ \\
\hline Ethylène glycols & + & + \\
\hline Styrène & + & +- \\
\hline $\begin{array}{l}\text { Hydrocarbures } \\
\text { aromatiques }\end{array}$ & + & $?$ \\
\hline Formaldéhyde & + & - \\
\hline Hypochlorite & + & - \\
\hline
\end{tabular}

non exposés $(\mathrm{n}=1020)$, exposés $(\mathrm{n}=1060)$ aux pesticides organochlorés / organo-phosphorés en Inde, Rupa et al. [102] ont rapportés une réduction de la proportion d'hommes féconds, une augmentation du taux de fausses-couches, de morts néonatales, de malformations chez les exposés. De Lock et al. dans une étude récente, montrent un délai nécessaire pour concevoir allongé chez les travailleurs exposés avec une augmentation de la fréquence de l'infécondité plus élevée dans le groupe fortement exposé comparé au groupe faiblement exposé [23]. Par contre une étude du spermogramme et de la chromatine des spermatozoïdes, menée dans le cadre du projet Asclepios [56], chez 161 exposés et 87 non exposés aux pesticides dans leur travail n'a pas mis en évidence de modifications du spermogramme entre avant et après l'utilisation des pesticides. Le pourcentage de cellules à chromatine intacte décroit significativement après l'utilisation des pesticides, mais les auteurs précisent que cette différence est dans les limites de variation du test. De plus, il semblerait exister un effet si la durée d'utilisation des pesticides est importante mais cela reste à démontrer.

Plus proche de nous l'étude menée, avant et après la période d'utilisation chez 25 viticulteurs Alsaciens par Thonneau et al. [112] ne montre pas d'altération de la concentration du nombre de spermatozoïdes ni du pourcentage de mobiles. Par contre il existe une tendance à la diminution de la numération et de la mobilité lorsque la quantité de produits utilisés est importante. Au delà de l'étude des caractéristiques spermatiques, l'intérêt de cette étude est de tenter de mesurer l'exposition par le dépistage des pesticides dans les cheveux : aucun des hommes n'avaient de pesticides dans les cheveux avant et 9 sur 25 en avaient après la période d'utilisation.

Dioxines et dérivés : familles de 75 produits, la plus connue est la 2,3,7,8 TCDD, l'origine de ces produits est secondaire à l'activité industrielle essentiellement des composés chlorés. La dioxine comme le DTT et les PCB a une forte affinité pour les corps gras et s'y accumule. On la retrouve comme contaminant de divers herbicides mais également sous forme de traces dans le sol, l'alimentation, l'eau. L'action délétère est connue chez l'animal y compris lors d'une exposition du mâle in utero à des doses très faibles [94]. Les études concernant la reproduction dans l'espèce humaine, réalisée chez des travailleurs exposés ou chez des vétérans du Vietnam après utilisation du célèbre agent orange, sont contradictoires.

Polychlorobiphényls (PCBS) : famille d'environ 200 produits largement utilisés, lipophiles. Chez des hommes exposés à l'Arochlor 
1260 il n'a pas été rapporté d'effet sur les anomalies du sperme ou la fertilité [27, 28]. L'épichlorohydrin est un produit également largement utilisé dans l'industrie des résines, de la glycérine, des insecticides et des plastiques. Chez l'homme, il n'y a pas d'effet démontré sur la spermatogenèse mais cela dans des études où le nombre de perdus de vue était important.

Métaux : le cadmium, s'il cause des lésions chez l'animal, ne semble pas être responsable chez l'homme de stérilité mais les études sont peu nombreuses [43, 77]. Le manganèse semble affecter la fertilité chez les travailleurs modérément exposés [69] alors que cela n'est pas mis en évidence dans une étude plus récente mais avec une exposition plus faible [43]. Par contre, il semble que ce métal puisse être à l'origine de dysfonction sexuelle mais chez des hommes hautement exposés, ceci probablement en rapport avec des atteintes neurologiques $[100,80]$. Plusieurs études ont fait état d'effets délétères de l'exposition au plomb sur la production et la qualité des spermatozoïdes $[67,22,4,71]$ avec parfois une corrélation inverse entre la plombémie et les caractéristiques du sperme. Ces effets ainsi que les effets de l'exposition au plomb sur les étapes postconceptionnelles de la reproduction sont largement discutés dans une excellente revue récente de Tas et al.[110], ainsi que les effets de l'exposition au mercure et au bore.

Les solvants organiques sont très largement employés dans l'industrie mais également dans la vie quotidienne (du lave-vitre aux médicaments). Le Carbon disulfure employé dans l'industrie chimique a été responsable d'oligo-asthéno-tératospermie chez des hommes exposés qui avaient également d'autres effets néfastes (polynévrites, dysfonction sexuelle) $[64,66]$.

La toxicité des éthers de glycol très largement employés, est actuellement beaucoup discutée. Néanmoins il apparaît opportun de considérer l'exposition aux éthers de glycol à risque pour la reproduction chez l'homme [110]. La toxicité d'autres produits comme les hydrocarbures dont le styrène n'est pas démontrée chez l'homme ou est en cours de discussion [110].

\section{FACTEURS PHYSIQUES}

\section{Radiations ionisantes}

La sensibilité de la spermatogenèse aux radiations ionisantes est connue depuis les célèbres travaux de Rowley et al. [101] sur les prisonniers "volontaires" américains. Une dose minime de 0,15 Gy $(150 \mathrm{mSv})$ est suffisante pour entraîner une oligospermie et une dose de 0,3 Gy une azoospermie dans les 50 à 60 jours qui suivent. La toxicité dépendant de la dose reçue est majorée par le fractionnement de la dose. Le testicule peut être soumis à une irradiation externe mais également à une irradiation due à une contamination interne. En effet il semble actuellement admis que des radionucléides peuvent être retrouvés dans les tubes séminifères notamment au niveau des cellules germinales transportées notamment par le système fer/transferrine pour certains d'entre eux [revue : 53]. Les radiations peuvent provoquer une mort cellulaire ou un grand nombre de lésions avec parfois des atteintes de l'ADN qui peuvent poser le problème de l'atteinte de la descendance. Chez des techniciens radiologues un effet délétère sur la fertilité avait été noté avec un effet dose dépendant et les mesures de radioprotection diminuaient le taux d'infertilité [60].

Les liens entre risque de fausses couches, prématurités et exposition aux radiations sont discutés, positifs pour deux études [9, 104], négatifs pour deux autres études [73, 78].

En Grande-Bretagne, Gardner et al. [42] avaient, en 1990, mis en évidence une relation entre exposition du père aux radiations $(\geq 100$ $\mathrm{mSv}$ en dose totale) et le risque d'avoir une leucémie $(\mathrm{RR}=8.38$ [1.35 à 51.9]) ou une leucémie et un lymphome non Hodgkinien $(\mathrm{RR}=8.59$ [1.4 à 52.0]) chez le jeune enfant. Lorsque la dose reçue dans les 6 mois avant la conception était de $10 \mathrm{mSv}$ ou plus le risque relatif était également augmenté $(\mathrm{RR}=6.82[1.46$ à 31.86]). Cette étude posait le problème d'une irradiation du père en milieu professionnel (industrie nucléaire) et pathologie chez l'enfant. D'autres travaux n'ont pas retrouvé de tels résultats et une étude plus large menée par Doll contredisait l'hypothèse de Gardner et $a l$. attribuant les résultats observés à d'autres 
causes possibles [25]. Plus récemment, l'étude de Viel montrant une augmentation de la fréquence des leucémies chez l'enfant autour du site de La Hague dans notre pays, relance le débat [95].

La catastrophe de Tchernobyl a provoqué l'irradiation de millions de personnes avec pour certaines d'entre elles de fortes expositions. Gorpinchenko et al. retrouvent chez les nettoyeurs, dans les années qui suivent l'irradiation, une fréquence élevée d'altérations quantitatives $(42,4 \%$ présentant une oligospermie ou une azoospermie) et qualitatives $(52,6 \%$ présentant une asthénospermie ou une tératospermie) de la spermatogenèse [47]. 7 à 9 ans après l'accident l'étude de 70 hommes exposés à en moyenne $0,16 \mathrm{~Gy}$ et celle de 20 non exposés montre une augmentation de la tératospermie chez les exposés [46]. Par ailleurs le groupe de Fischbein et Bartow va étudier le spermogramme et l'ultrastructure des spermatozoïdes de 18 hommes non exposés et 18 qui ont été exposés quelques minutes en zone irradiée ou qui avaient travaillé durant 8 mois dans une zone de $30 \mathrm{~km}$. Une diminution de la mobilité est retrouvée chez les exposés, et en microscopie électronique ils mettent en évidence une diminution du pourcentage de spermatozoïdes ayant un noyau intact et une augmentation des têtes irrégulières et ceci dix ans semble-t-il après l'irradiation [36].

En accord avec le résultat des études faites chez les rongeurs, Dubrova et al. retrouvent une augmentation des mutations sur les cellules germinales de la descendance des populations hautement contaminées par les rejets de Tchernobyl, comparés à une population non contaminée [26]. A l'inverse, une étude réalisée sur les descendants des japonais soumis à l'irradiation aiguë de la bombe atomique ne retrouvait pas d'induction de mutations [63].

Plus prés de nous une étude de type cas $(n=60)$ témoin ( $\mathrm{n}=165)$ menée chez des miltaires de la région de Brest met en évidence, après analyse multivariée ayant intégré les facteurs de risque pour la fertilité chez l'homme et chez la femme, un risque augmenté de consulter pour infécondité si l'homme est exposé à la chaleur (OR: 5,4 [1,8-16,4]) ou aux radiations (OR : 2,9 $[1,3-6,3])$. Ces expositions étant dûes au travail dans les sous marins en ce qui concerne les radiations, et aux professions de cuisinier, sous marinier en ce qui concerne la chaleur [116]. D'autres études, notamment de type exposé/ non exposé, intégrant la mesure de l'exposition sont urgentes pour infirmer ou confirmer l'effet de l'exposition professionnelle aux radiations sur la spermatogenèse.

Par ailleurs, si les effets des radiations sur la spermatogénèse sont connus, de nombreuses études restent à mener pour préciser l'impact de l'irradiation du père (contamination externe/interne, durée aiguë/chronique...) sur sa descendance.

\section{Radiations non ionisantes}

Une étude avait montré des altérations du sperme et des troubles de la libido chez des travailleurs exposés à des micro-ondes depuis en moyenne 8 ans [65] avec un retour à la normale chez les $2 / 3$ d'entre eux en trois mois après l'arrêt de l'exposition, mais sans information précise sur les circonstances de l'exposition.

L'exposition aux champs électromagnétiques est fréquente dans la société moderne. La proportion d'hommes exposés aux champs électromagnétiques (industrie électrique) a été retrouvée augmentée chez les patients oligospermes ou azoospermes dans une étude [11] mais pas dans une autre [75]. Cependant il a été récemment rapporté une diminution du nombre d'enfant et du sex ratio chez des travailleurs exposés à de fortes intensités comparés à ceux exposés à des champs d'intensité moindre [62] et deux fois plus de difficulté à concevoir un enfant ainsi qu'un sex ratio diminué et une augmentation des anomalies congénitales chez des travailleurs exposés comparés à des non exposés [89].

\section{Chaleur}

Les effets délétères de l'hyperthermie sur la spermatogénèse sont actuellement parfaitement admis. Nous avons antérieurement démontré, dans notre groupe, qu'une faible augmentation de la température testiculaire, lors d'une cryptorchidie expérimentale induite durant les heures d'éveil chez l'homme, entrainait une altération quantitative [81] et qualitative [82] de la spermatogenèse. Ces modifica- 
tions avaient pour conséquence une absence de fertilité et nous avons pu proposer cette méthode comme un moyen de contraception masculine [83]. Le lecteur intéressé par les relations entre température et spermatogenèse / fertilité pourra se reporter à une synthèse que nous avons récemment publiée notamment dans cette même revue $[84,13]$.

L'exposition à la chaleur peut chez l'homme se faire sous la forme de deux modalités : une exposition de forte intensité le plus souvent en milieu professionnel et une exposition de faible intensité.

\section{Exposition intense à la chaleur : exposi- tions professionnelles.}

Plusieurs professions sont potentiellement exposées à la chaleur : soudeurs, céramistes, sidérurgistes, cuisiniers, pompiers... Réalisant une étude cas témoins, Rachootin et Olsen retrouvent une augmentation significative de la fréquence de l'exposition à la chaleur chez les patients inféconds ou ayant des anomalies du sperme comparés aux témoins [98]. La soudure expose l'homme à des gaz issus du procédé de soudure et à une augmentation importante de la température. Bonde retrouve chez des soudeurs uniquement exposés à la température (hotte aspirante pour les gaz) une augmentation du nombre de spermatozoides morphologiquement anormaux avec une récupération environ 4 semaines après l'arrêt [7].

D'autres études suggèrent un lien entre exposition à la chaleur et altération des paramètres du sperme, retard à la conception chez des ouvriers de l'industrie céramique comparé à des hommes non exposés [35] ainsi qu'une augmentation du risque relatif de faible poids de naissance et prématurité [103] et de faussecouche [61] chez les travailleurs exposés à la chaleur.

Si l'exposition professionnelle à une température élevée apparaît comme un facteur de risque pour la spermatogénèse / la fertilité de nombreuses études restant encore à faire pour quantifier l'exposition et analyser l'effet dose.

\section{MODES DE VIE}

S'il est encore prématuré de mettre en relation les changements du mode de vie de ces der- nières cinquante années avec la décroissance de la spermatogenèse observée dans certaines régions il apparaît évident que certaines modalités de notre mode de vie représentent un risque pour la fonction exocrine du testicule.

\section{Exposition modérée à la chaleur : habillement / position}

L'habillement ou la posture peuvent influencer la température scrotale chez l'homme [10]. Si certains accusent la mode des jeans serrés d'être néfaste pour la spermatogénèse, une étude particulièrement intéressante à été réalisée par Tiemessen et al. [113] qui fait porter à des hommes volontaires soit des sous-vêtements serrés (slips) soit des sous-vêtements lâches (caleçons) au cours d'un essai croisé. Le port des sous-vêtements serrés entraîne une diminution significative du nombre de spermatozoïdes (46 [14-119] vs 89 [17-173] x 106/ml) ainsi qu'une diminution du nombre de spermatozoïdes mobiles $(17[0,6-82]$ vs $53[0,8-101] \mathrm{x}$

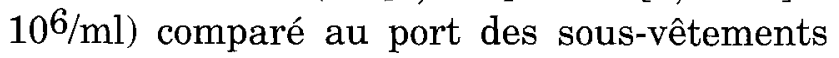
plus lâches. Dans le même sens mais dans une étude méthodologiquement moins démonstrative, Parazzini et al. [93] étudiant 218 inféconds, trouve 2,5 fois plus d'hommes ayant des habits serrés chez les oligo-asthénospermes comparés aux normospermes $(\mathrm{OR}=2.5[1.5$ à 5.2]).

Thonneau et al. avec notre groupe [111] retrouveut une augmentation du délai nécessaire pour concevoir si l'homme est exposé professionnellement à la chaleur ou s'il est assis plus de 3 heures dans un véhicule. En analyse multiparamétrique le risque de non conception dans les trois premiers mois est multiplié par 4 $(\mathrm{OR}=4.0$ [1.4 à 11.4]) si l'homme est assis plus de 3 heures, par 1,7 (OR = 1.7 [1.0 à 2.8]) s'il est exposé à la chaleur et par $1,6(\mathrm{OR}=1.6[1.0$ à 2.6]) si la femme fume.

Par ailleurs l'étude de Figa-Talamenca et al. [34] montre une atteinte morphologique des spermatozoïdes chez des hommes ayant la profession de taxi comparés à des témoins.

Si l'hypothése d'une augmentation de la température scrotale était avancée pour expliquer les modifications du délai nécessaire pour concevoir ou les perturbations du spermogramme en cas de position assise prolongée dans un véhicule il restait à la démontrer. Nous avons 
donc entrepris l'étude de la température scrotale chez neuf hommes volontaires lors de la conduite d'un véhicule. Après pose de sondes thermiques sur le scrotum en regard de la face antérieure de chaque testicule un enregistrement de la température scrotale et de la température ambiante est programmée toutes les deux minutes. Ces volontaires vont ensuite marcher et se tenir debout dans un bus durant 40 minutes le temps d'arriver à un garage qui leur fournira une voiture avec laquelle il vont effectuer 160 minutes de conduite. Les résultats montrent une augmentation rapide de la température scrotale de $34,2^{\circ} \mathrm{C}$ à $35,5^{\circ} \mathrm{C}$ dans les premières 20 minutes de la conduite suivie dans les 2 heures d'une augmentation moins rapide pour atteindre $36,2^{\circ} \mathrm{C}$ [15]. Ainsi l'hypothèse évoquée antérieurement vient d'être vérifiée et comme nous l'avions suggéré antérieurement le temps passé en position de conduite pourrait participer au débat sur les modifications de la spermatogenèse décrites chez l'homme [12].

\section{Stress}

Les relations entre stress et spermatogenèse ou fertilité masculine sont de nos jours peu connues bien que des études ont pu montrer des différences entre catégories professionnelles. Une étude récente [33] chez 164 hommes avec pour 157 deux examens de sperme n'a pas mis en évidence de lien entre la qualité du travail ("stressant ou non stressant"), le divorce ou la séparation et les caractéristiques du sperme. Par contre les hommes ayant eu un stress important (décès d'un proche dans la famille) dans les trois mois précédents présentaient une altération de la mobilité et de la forme des spermatozoïdes. Le tremblement de terre de Kobe au Japon (1995) a constitué un stress notable pour les habitants, 27 patients étaient suivis à ce momentlà et une diminution du pourcentage de spermatozoïdes mobiles a été notée seulement chez ceux qui avaient eu leur maison détruite avec une récupération dans les 2 à 9 mois [40].

\section{Tabac}

La littérature concernant les relations entre tabac et spermatogenèse est abondante sans que les conclusions soient définitives.
Cependant, une méta analyse réalisée par Vine et al. [119] reprenant les études publiées entre 1966 et 1992 conclut que les fumeurs ont une concentration en spermatozoïde 13 à $17 \%$ plus basse que les non fumeurs. Par ailleurs, étudiant 88 fumeurs et 40 non fumeurs il a été retrouvé une corrélation inverse entre le nombre de cigarettes par jour, le nombre d'années de tabagisme, les taux de conitine (métabolite de la nicotine) et la concentration, la mobilité et le nombre total de spermatozoïdes [120].

Vine émet audacieusement l'hypothèse d'un lien entre le tabac et la décroissance séculaire de la spermatogenèse avec une diminution de la consommation $\mathrm{du}$ tabac ces dernières années et une remontée de la numération (retrouvée suivant un modèle mathématique) [118].

Par ailleurs si la relation entre tabagisme du père avant la conception et risque de pathologie chez l'enfant est discutée, il est intéressant de rapporter l'étude de Bu-Tian Ji et al. [17] qui met en évidence une augmentation du risque relatif de faire une leucémie lymphoïde aigue (OR: $3,8[1,3-12,3]$ ), un lymphome (OR: $4,5[1,2-16,8]$ ), un cancer (OR: 1,7 [1,2-2-5]) chez l'enfant, lors de l'exposition paternelle préconceptionnelle. Cette étude a été réalisée dans une province chinoise ou le pourcentage de femme qui fument était inférieur à $1 \%$. Montrant que l'exposition d'au moins cinq ans avant la conception peut entraîner une augmentation du risque, elle pourrait souligner l'impact d'un facteur exogène sur le spermatozoïde et la descendance.

\section{L'alcool}

La consommation chronique importante d'alcool entraînant des perturbations hépatiques va modifier le profil hormonal des patients avec des altérations éventuelles de la fonction de reproduction. $\mathrm{Si}$, dans une étude multicentrique européenne récente, aucune relation n'a été mise en évidence entre le délai nécessaire pour concevoir et la consommation d'alcool par l'homme ou la femme, d'autres études montrent une action de l'alcool sur la production de sperme.

Ainsi, étudiant 38 alcooliques asymptoma- 
tiques et 19 contrôles, il apparaît un lien entre le nombre d'années d'alcoolisme et les caractéristiques du sperme en analyse multivariée, 39 $\%$ des alcooliques ont une diminution du nombre de spermatozoïdes [117]. Dans le même sens une étude de tissu testiculaire lors des autopsies met en évidence une altération de la spermatogenèse chez des hommes ayant une consommation modérée ou élevée d'alcool [92].

\section{Le sport}

Si l'effet du sport de haut niveau et notamment les sports à efforts soutenus sont connus pour perturber la fertilité féminine, peu d'études ont été réalisées chez l'homme. Lucia et al. vont étudier des athlètes de très haut niveau et notamment 12 cyclistes professionnels (certains participant au Tour de France), 9 triathlètes et 10 marathoniens, et ce, avant, pendant et après la saison des compétitions. Aucune différence n'est mise en évidence en dehors d'une diminution du pourcentage de spermatozoïdes mobiles retrouvés chez les cyclistes et qu'ils attribuent soit à des microtraumatismes soit à des problèmes de microtraumatismes ou d'hyperthermie locale, le système endocrine n'étant en rien modifié [74].

\section{Alimentation}

Des produits comme les métabolites du DDT, les PCBs ont pu être retrouvés chez l'homme sans relation avec la profession [105] et l'hypothèse d'une contamination large a été évoquée notamment par celle de la chaîne alimentaire. Jensen et al. [57] vont comparer un groupe d'hommes $(\mathrm{n}=58)$ "adeptes de l'agriculture biologique" à un groupe témoin $(\mathrm{n}=141)$.

La concentration de spermatozoïdes est plus haute chez les hommes adeptes de l'agriculture biologique, et en analyse multiparamétrique incluant les saisons, l'âge, le délai d'abstinence, cette concentration est $43 \%$ plus haute.

Bien que l'alimentation puisse jouer un rôle ici, d'autres hypothèses doivent être évoquées comme la localisation urbaine ou rurale et le mode de vie en général (existe-t-il moins de stress chez les adeptes de l'agriculture biologique?).

Dans une autre étude danoise, 256 fermiers ont été classés en trois groupe suivant leur pratique alimentaire et notamment la consommation de fruits et légumes "biologiques". Leur ingestion de produits phytosanitaires a été calculé à partir de leur alimentation. Il existe une diminution du pourcentage de spermatozoïdes de forme normale chez ceux qui ne mangent jamais de fruits et légumes biologiques comparés à ceux dont l'ingestion produits biologiques est élevée. A partir de leur dose quotidienne absorbée l'étude des corrélations entre la quarantaine de pesticides et les caractéristiques spermiologiques a seulement mis en évidence une corrélation inverse entre 5 pesticides et la vitalité des spermatozoïdes [55].

\section{Pollution.}

Bien entendu la pollution est souvent incriminé pour expliquer l'augmentation de certaines pathologie comme les affections respiratoires. En ce qui concerne le champ de la reproduction l'étude de Sram et al. nous paraît intéressante [108]. Ces auteurs, dans le cadre d'un vaste programme multidisciplinaire, ont comparé deux régions tchèques : une très industrielle dans le nord ou la forêt a été pratiquement détruite par la pollution et ou une augmentation des affections respiratoires et des cancers semblent avoir été constatée, à une région sans activité industrielle dans le sud. Ils ont mesuré certains indicateurs comme la concentration en dioxide de soufre, oxyde d'azote, particules aériennes, hydrocarbures polycycliques et autres polluants de l'air. Leur étude a porté sur la prématurité, les retards de croissance, la présence d'adduits sur le placenta... En ce qui concerne la spermatogenèse ils mettent en évidence un lien entre la morphologie du spermatozoïde et la pollution de l'air. L'analyse de l'aneuploïdie du spermatozoïde montre une augmentation de la disomie du chromosome $\mathrm{Y}$ lors de la saison la plus polluée.

\section{CONCLUSION}

1) La réalité d'une décroissance de la spermatogenèse dans certaines régions ainsi que la mise en évidence d'une augmentation des atteintes de l'appareil de reproduction tant chez l'homme que dans la faune sauvage font suspecter une influence de l'environnement 
sur l'appareil de reproduction chez le mâle. Cette suspicion est largement fondée à la lumière des exemples historiques, d'exposition à des substances durant la vie adulte ou la vie in utero (DES, DBCP, gossypol) mais également à la lumière des accidents survenue dans la faune sauvage (lac Apopka). Authentifier les responsables peut-être facile si l'effet est important et rapide, mais, bien plus difficile si les effets sont plus minimes, s'inscrivant dans une action à long terme, ou, s'ils sont dus à des causes multiples. L'hypothèse des modificateurs ou perturbateurs endocriniens est une piste qui apparaît des plus sérieuses. Pour certains nous baignons actuellement dans une "mer de molécules œstrogènes like" et nous savons que certains produits chimiques ont des actions anti-androgènes. Il est particulièrement intéressant de souligner que ces molécules peuvent avoir une synergie très puissante. Au-delà de l'hypothèse des modificateurs endocriniens nous venons de voir dans ce travail que d'autres pistes méritent des explorations approfondies et au-delà des expositions professionnelles [110, 14] il s'agit du mode de vie des populations. Il nous semble indispensable, afin d'étudier les différences régionales concernant la spermatogenèse, de rechercher les différences dans les dénominateurs communs que sont par exemple l'eau, l'air, le mode de vie.

2) Il est important de noter qu'un facteur exogène peut agir de différentes façons sur la spermatogenèse. Il peut provoquer un effet direct avec pour conséquences une altération des paramètres spermatiques ou du spermatozoïde (contenu de la cellule et notamment le noyau). Mais il peut être à l'origine de conséquences comme l'allongement de délai nécessaire pour concevoir (DNC), une altération du développement embryonnaire ou de la descendance. Il est vraisemblable qu'il existe un continuum qui va de l'action sur la spermatogenèse jusqu'à la descendance et son développement. Par contre la visibilité des ces effets peut être tout à fait partielle (Figure 1).

3) Envisager la problématique des liens entre environnement et spermatogenèse doit faire poser la question : quelles études, quels outils ? Les études de type exposés/non exposés semblent plus performantes que les études cas/témoins. Il faut souligner que la mesure d'exposition est le "talon d'Achille" des études épidémiologiques sur l'environnement et une attention particulière doit être portée sur ce point. En ce qui concerne les outils si l'on utilise couramment la mesure du DNC, la fréquence de survenu d'une fausse couche spontanée, les paramètres du spermogramme, les tests mesurant l'atteinte du génome du spermatozoïdes doivent être développés (FISH, Comet Assay, Tunel Assay, recherche d'adduits). Dans ce sens, en association avec notre groupe l'équipe du Pr. Leszkowicz de l'ENSAT de Toulouse vient de mettre en évidence pour la première fois la présence d'adduits dans les cellules du sperme humain.

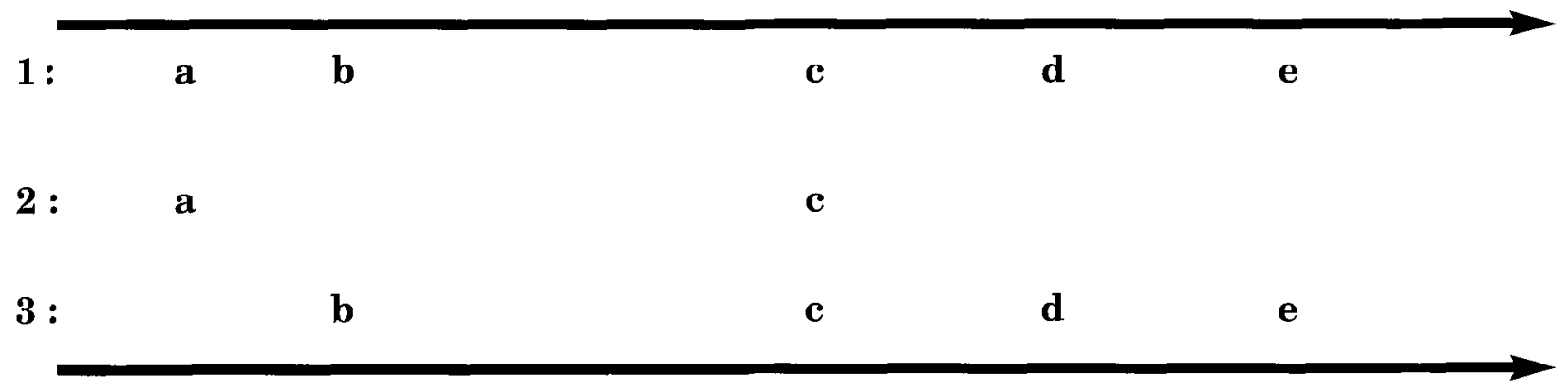

Figure 1 : Effets d'un facteur exogène : les modifications peuvent se traduire à plusieurs niveaux : a: paramètres spermatiques, $b$ : spermatozoüde (noyau), c : délai nécessaire pour concevoir, $d$ : développement embryonnaire, $e$ : descendance. Exemples: en 1: modifications à tous les niveaux, en 2 : action visible sur spermogramme et délai nécessaire pour concevoir, en 3 : action non visible sur le spermogramme mais ayant des conséquences sur les autres événements. 
4) Les liens entre environnement et spermatogenèse pourraient être étudiés à la lumière de facteurs génétiques. Ceci est facilement illustré par l'étude récente de C. Infante-Rivard et al.[54]: l'utilisation par la mère de pesticides contre les mites et les cafards durant la grossesse augmente de façon non significative le risque pour l'enfant de faire une leucémie lymphoïde aiguë (OR: 1,4 [0,15-12,82]). Si l'analyse est menée en tenant compte du polymorphisme génétique de la mère et notamment celui pour le système enzymatique cytochrome $\mathrm{P} 450$, le risque devient significatif (OR: 5,02 [1,00-29,09]. Les études ultérieures devraient prendre en compte les polymorphismes génétiques qui peuvent modifier les processus de détoxification, le métabolisme ou la résistances de l'organisme aux agents exogènes.

Tous les aspects soulevés ici soulignent l'indispensable nécessité d'une recherche pluridisciplinaire associant des cliniciens, des épidémiologistes, des généticiens, des médecins du travail, des toxicologues, des médecins et biologistes de la reproduction, des écologistes scientifiques et des chercheurs des sciences fondamentales (ex : biochimiste) ou appliquées (ex. : sociologues, urbanistes). Une telle recherche doit être indépendante et libre et doit pouvoir disposer de moyens ce qui implique une réelle volonté politique.

Dans l'attente d'une réponse à toutes ces questions, il nous semble utile, eu égard à la fragilité de la spermatogenèse et au fait que le spermatozoïde est une cellule facile à obtenir sans effraction, de proposer "le sperme comme indicateur de santé publique" en accord avec A. Spira.

Par ailleurs l'analyse de l'histoire du DBCP avec une toxicité connue chez l'animal en 1960 et une altération décrite chez les hommes exposés en 1977 devrait être riche d'enseignement et nous rappeler que tout cela aurait pu être évité si l'on avait tenu compte du "Principe de précaution".

Nous conclurons donc cette revue sur environnement et spermatogenèse en rappelant ce principe de précaution qui peut se traduire très concrètement :

- par une réelle action de prévention en milieu professionnel,
- par l'évitement d'un facteur de risque découvert lors des consultations pour infécondité,

- par la non-utilisation de substances n'ayant pas fait la preuve de leur innocuité dans les études toxicologiques chez l'animal.

Il serait souhaitable par ailleurs de créer des centres de spermovigilance où pourraient être signalés et étudiés les effets de facteurs exogènes sur la spermatogenèse et le spermatozoïde.

Ceci implique une réelle réflexion de tous les acteurs de notre Société.

Remerciement : l'auteur remercie très vivement et chaleureusement les Drs R. Mieusset, J. Auger, B. Jégou, $P$. Thonneau ainsi que madame le Pr. Leszkowicz pour les riches échanges sur le sujet. Remerciement à Marie-Claude Bacou pour la préparation du manuscrit.

\section{RÉFÉRENCES}

1. ADAMI H., BERGSTRÖM R., MÖHNER M. et al. : Testicular cancer in nine Northern European countries. Int. J. Cancer, 1994, 59 : 33-38.

2. ANDERSON T., FORLIN L., HARIG I., LARSSON A.: Physiological distrubances in fish living in coastal water polluted with bleached kraft mill effluents. Can. J. Fish Aquat. Sci., 1988, 45 : 1525-1536.

3. ANDONIAN R.W., KESSLER R. : Transplacental exposure to diethylstilbestrol in men. Urology, 1976, $13: 276-279$.

4. ASSENATO G., PACI C., BASER M.E. et al. : Sperm count suppression without endocrine dysfunction in lead exposed men. Arch. Environ. Health, 1987, 42 : 124-127.

5. AUGER J., KUNSTMANN J.M., CZYGLIK F., JOUANNET P. : Decline in semen quality among fertile men in Paris during the past 20 years. New Engl. J. Med., 1995, 332 : 281-285.

6. BERGERON J.M., CREWS D., MCLACHLAN J.A. : PCBs as environmental estrogens : turtle sex determination as a biomarker of environmental contamination. Environ. Health Perspect., 1994, 102 : 780-781.

7. BONDE J.P. : Semen quality in welders exposed to radiant heat. Br. J. Ind. Med., 1992, 49 : 5-10.

8. BOSTOFTE E., SERUP J., REBBE H. : Has the fertility of Danish men declined through the years in terms of semen quality? A comparison of semen qualities between 1952 and 1972. Int J. Fertil., 1983, 28: 91-95. 
9. BOUE J., BOUE A., LAZAR P. : Retrospective and prospective epidemiologic studies of 1500 karyotyped spontaneous abortions. Teratology, 1975, 12 : 11-26.

10. BRINDLEY G.S. : Deep scrotal temperature and the effect on it of clothing, air temperature, activity, posture and paraplegia. Brit. J. Urol., 1982, 54 : 49-55.

11. BUIATTI E., BARCHIELLI A., GEDDES M. et al. : Risk factors in male fertility : a case-control study. Arch. Environ. Health, 1984, 39 : 266-270.

12. BUJAN L., MANSAT A., PONTONNIER F., MIEUSSET R. : Time series analysis of sperm concentration in fertile men in Toulouse, France, between 1977 and 1992. Brit. Med. J., 1996, 312 : 471472.

13. BUJAN L., MIEUSSET A. : Température et fertilité masculine. Contracept. Fertil. Sex., 1996, 24: 429438.

14. BUJAN L., MIEUSSET R., MANSAT A., PONTONNIER F. : Conditions de travail, spermatogénèse et fertilité masculine. Arch. Mal. Prof., 1988, $49: 97-102$.

15. BUJAN L., DAUDIN M., CHARLET J.P., THONNEAU P., MIEUSSET R. : Increase in scrotal temperature in car drivers. Hum. Reprod., 2000, 15 : $1355-1357$.

16. BURLINGTON H., LINDEMAN : Effect of DDT on testes and secondary characters of white leghorn cockerels. Proc. Soc. Exp. Biol. Med., 1950, 74 : 48-51.

17. BU-TIAN J.I., SHU X.O., LINET M.S. et al. : Paternal cigarette smoking and the risk of chilhood cancer among offspring of nonsmoking mothers. J. Natl. Cancer Inst., 1997, 89 : 238-244.

18. CAMPBELL D.M., WEBB J.A., HARGREAVE, T.B. : Cryptorchidism in Scotland. Br. Med. J., 1987, 295 : 1237-1238.

19. CARLSEN E., GIWERCMAN A., KEIDING N., SKAKKEBAEK N.E. : Evidence for decreasing quality of semen during past 50 years. Brit. Med. J., 1992, $305: 609-613$.

20. COLBORN T. : Developmental effects of endocrinedisrupting chemicals in wildlife and humans. Environ. Health. Perspect., 1993, $101: 378-384$.

21. COOPER R.L., KARLOCK R.J. : Endocrine disruptors and reproductive development : a weight of evidence overview. J. Endocrinol., 1997, 152 : 159-166.

22. CULLEN M.R., KAYNE R.D., ROBINS J.M. : Endocrine and reproductive dysfunction in men associated with occupational inorganic lead intoxication. Arch. Environ. Health., 1984, 39 : 431-440.

23. DE COCK J., WESTVEER K., HEEDERIK D., TE VELDE E., VAN JOOIJ R. : Time to pregnancy and occupational exposure to pesticides in fruit growers in The Netherlands. J. Environ. Med., 1994, 51 : 693699.

24. DIECKMANN W.J., DAVIS M.E., RYNKIEWICZ
L.M., POTTINGER R.E. : Does administration of diethylstilbestrol during pregnancy have therapeutic value ? Am. J. Obstet. Gynecol., 1953, 66 :102.

25. DOLL R., EVANS H.J., DARBY S.C. : Paternal exposure not to blame. Nature, 1994, $367: 678-680$.

26. DUBROVA Y.E., NESTEROV V.N., KROUCHINSKY N.G. et al. : Human minisatelite mutation rate after the Tchernobyl accident. Nature, 1996, $380: 683-686$.

27. EMMETT E.A., MARONI M., JEFFERYS J., SCHMITH J.M., LEVIN B.K., ALVARES A. : Studies of transformer repair workers exposed to PCBs. II. Results of clinical laboratory investigations. Am. J. Ind. Med., 1988, 14 : 47-62.

28. EMMETT E.A., MARONI M., SCHMITH J.M., LEVIN B.K., JEFFERYS J. : Studies of transformer repair workers exposed to PCBs. I. Study design, PCB concentrations, questionnaire, and clinical examination results. Am. J. Ind. Med., 1988, 13 : 415427.

29. EWERTZ M., HOLMBERG L., KARJALAINEN S., TRETLI S., ADAMI H.O. : Incidence of male breast cancer in Scandinavia, 1943-1982. Int. J. Cancer, $1989,43: 27-31$.

30. FACEMIRE C.F., GROSS T.S., GUILLETTE L.J.J. : Reproductive impairment in the Florida panther : nature or nurture. Environ. Health Perspect., 1995, 103 (suppl. 4) : 79-86.

31. FARROW S. : Falling sperm quality : fact or fiction ? Brit. Med. J., 1994, 309 : 1-2.

32. FÉDÉRATION FRANÇAISE DES CECOS, AUGER J., JOUANNET P. : Evidence for regional differences of semen quality among fertile french men. Hum. Reprod., 1997, $12:$ 740-745.

33. FENSTER L., KATZ D.F., WYROBEK A.J., PIEPER C., REMPEL D.M., SWAN S.H. : Effect of psychological stress on human semen quality. J. Androl., 1997, $18: 194-202$.

34. FIGA-TALAMANCA I., CINI C., VARRICCHIO G.C. et al. : Effects of prolonged autovehicle driving on male reproduction function : a study among taxi drivers. Am. J. Ind. Med., 1996, 30 : 750-758.

35. FIGA-TALAMANCA I., DELL'ORCO V., PUPI A. $e t$ al. : Fertility and sperm quality of workers exposed to high temperatures in ceramics industry. Reprod. Toxicol., 1992, $6: 517-523$.

36. FISCHBEIN A., ZABLUDOVSKY N., ELTES F., GRISCHENKO V., BARTOOV B. : Ultramorphological sperm characteristics in the risk assessment of health effects after radiation exposure among salvage workers in Chernobyl. Environ. Health Perspect., 1997, 105 (suppl. 6) : 1445-1449.

37. FORMAN D., MOLLER H. : Testicular cancer. Cancer Surv., 1994, 19/20 : 323-341.

38. FRY D.M., TOONE C.K. : DDT-induced feminization of gull embryos. Science, 1981, $213: 922-924$. 
39. FRY D.M., TOONE C.K., SPEICH S.M., PEARD R.J.: Sex ratio skew and breeding patterns of gulls : demographic and toxicological considerations. Stud. Avian Biol., 1987, $10: 26-43$.

40. FUKUDA M., FUKUDA K., SHIMIZU T., YOMURA W., SHIMIZU S. : Kole earthquake and reduced sperm motility. Hum. Reprod., 1996, 11 : 1244-1246.

41. GARCIA-RODRIGUEZ J., GARCIA-MARTIN M., NOGUERAS-OCANA M. et al. : Exposure to pesticides and cryptorchidism : geographical evidence of a possible association. Environ. Health Perspect., 1996, $104: 1090-1095$.

42. GARDNER M.J., SNEE M.P., HALL A.J., POWELL C.A., DOWNES S. TERRELL J.D. : Results of casecontrol study of leukaemia and lymphoma among young people near Sellafield nuclear plant in West Cumbria. Brit. Med. J., 1990, 300 : 423-434.

43. GENNART J.P., BUCHET J.P., ROELS H., GHYSELEN P., CEULEMANS E., LAUWERYS R. : Fertility of male workers exposed to cadmium, lead, or manganese. Am. J. Epidemiol., 1992, 135 : 12081219.

44. GILL W.B., SCHUMACHER G.F.B., BIBBO M. : Pathological semen and anatomical abnormalities of the genital tract in human male subjects exposed to diethylstilbestrol in utero. J. Urol., 1977, $117: 477$ 480 .

45. GILL W.B., SCHUMACHER G.F.B., BIBBO M., STRAUS F.H.I., SCHOENBERG H.W. : Association of diethylstilbestrol exposure in utero with cryptorchidism, testicular hypoplasia and semen abnormalities. J. Urol., 1979, 122, 36-39.

46. GONCHAROV N.P., KATSIYA G.V., KOLESNIKOVA G.S. et al. : Endocrine and reproductive health status of men who had experienced short-term radiation exposure at Chernobyl. Int. J. Androl., 1998, 21 : 271276.

47. GORPINCHENKO I.I., BOYKO N.I. : Semen parameters of clean-up workers of chernobyl accident. Int. J. Androl., 1997, 20 (suppl. 1) : 63.

48. GUILLETTE L.J. Jr., CRAIN D.A., ROONEY A.A., PICKFORD D.B. : Organization versus activation : the rôle of endocrine-disrupting contaminants (EDCs) during embryonic development in wildlife. Environ. Health Perspect., 1995, 103 (suppl. 7) : 157164.

49. GUILLETTE L.J.J., GROSS T.S., MASSON G.R., MATTER J.M., PERCIVAL H.F., WOODMARD A.R. : Development abnormalities of the gonad and abnormal sex hormone concentrations in juvenile alligators from contaminated and control lakes in Florida. Environ. Health Perspect., 1994, 102 : 680-688.

50. GUZELIAN P.S. : Chlordecone poisoning : a case study in approaches for the detoxification of human exposed to environmental chemicals. Drug Metab. Rev., 1982, $13: 663-679$.
51. HENDERSON B.E., BENTON B., COSGROVE M. $e t$ al. : Urogenital tract abnormalities in sons of women treated with diethylstilbestrol. Pediatrics, 1976, 58 : 505-507.

52. HERBST A.L., ULFELDER H., POSKANZER D.C. : Adenocarcinoma of the vagina. Association of maternal stilbestrol therapy with tumour appearance in young women. New Engl. J. Med., 1971, 284 : 878881.

53. HOYES K.P., MORRIS I.D. : Environmental radiation and male reproduction. Int. J. Androl., 1996, 19: 199-204.

54. INFANTE-RIVARD C., LABUDA D., KRAJINOVIC M., SINNETT D. : Risk of childhood leukemia associated with exposure to pesticides and with gene polymorphism. Epidemiology, 1999, $10: 481-487$.

55. JULHER R.K., LARSEN S.B., MEYER O. et al. : Human semen quality in relation to dietary pesticide exposure and organic diet. Arch. Environ. Contam. Toxicol., 1999, $37: 415-423$.

56. LARSEN S.B., GIWERCMAN A., SPANO M., BONDE J.P., THE ASCLEPIOS STUDY GROUP : A longitudinal study of semen quality in pesticides spraying danish farmers. Reprod. Toxicol., 1998, 12 : 581-589.

57. JENSEN T.K., GIWERCMAN A., CARLSEN E., SCHEIKE T., SKAKKEBAEK N.E. : Semen quality among members of organic food associations in Zealand, Denmark. Lancet, 1996, $347: 1844$.

58. KELCE W.R., STONE C.R., LANS S.C., GRAY L.E., KEMPPAINEN J.A., WILSON E.M. : Persitent DDT metabolite p,p'-DDE is a potent androgen receptor antagonist. Nature, 1995, $375:$ 581-585.

59. KHARRAZI M., POTASHNIK G., GOLDSMITH J.R.: Reproductive effects of dibromochloropropane. Isr. J. Pestic. Sci., 1980, 10 : 403-406.

60. KITABAKAKE T., WATANABE T., SATO T. : Sterility in japanese radiological technicains. Tohobu. J. Exp. Med., 1974, 112 : 209-212.

61. KLINE J., STEIN Z., HATCH M., HUTZLER M., LAI A., PANTEL-SILVERMAN J. : Surveillance of Parental Employment and Spontaneous Abortion. Final report to the National Institute for Occupational Safety and Health. Contract No 210815002, National Institute for Occupational Safety and Health. Cincinnati, OH, 1982.

62. KNAVE B., GAMBERALE F., BERGSTROM S. et al.: Long-term exposure to electric fields : a cross-sectional epidemiologic investigation of occupationally exposed workers in high voltage substations. Scand. J. Work Environ. Health., 1979, 5 : 115.

63. KODAIRA M., SATOH C., HIYAMA K., TOYAMA K. : Lack of effects of atomic bomb radiation on genetic instability of tandem-repetitive elements in human germ cells. Am. J. Hum. Genet., 1995, 57 : 1275-1283.

64. LANCRANJAN I. : Alterations of spermatic liquid in 
patients chronically poisoned by carbon disulfide. Med. Lav., 1972, 63 : 123-125.

65. LANCRANJAN I., MAIGANESCU M., RAFAILA E., KLEPSCH I., POPESCU H.I. : Gonadic function in workmen with long term exposure to microwave. Health Phys., 1975, 29 : 381-383.

66. LANCRANJAN I., POPESCU H.I., KLEPSCH I. : Changes of the gonadic function in chronic carbon disulfide poisoning. Med. Lav., 1969, 60 : 566-571.

67. LANCRANJAN L., POPESCU H.I., GAVANESCU O., KLEPSCH I., SERBANESCU M. : Reproductive ability of workmen occupationally exposed to lead. Arch. Environ. Health, 1975, 30 : 396-401.

68. LANTZ G.D., CUNNINGHAM G.R., HUCKINS C., LIPSHULTZ L.I. : Recovery from oligospermia after exposure to dibromochloropropane. Fertil. Steril., 1981, $35:$ 46-53.

69. LAUWERYS R., ROELS H., GENET P., TOUSSAINT G., BOUCKAERT A., DE COOMAN S. : Fertility of male workers exposed to mercury vapors or to manganese dust : a questionnaire study. Am. J. Ind. Med., 1985, $7: 171-176$.

70. LERCHL A., NIESCHLAG E. Decreasing sperm counts ? A critical (re)view. Exp. Clin. Endocrinol. Diabetes, 1996, 104 : 301-307.

71. LERDA D. Study of sperm characteristics in persons occupationally exposed to lead. Am. J. Ind. Med., 1992, 22 : 567-571.

72. LETO S., FRENSILLI F.J. : Changing parameters of donor semen. Fertil. Steril., 1981, 36 : 766-770.

73. LINDBOHM M.L., HEMMINKI K., BONHOMME M.G. et al. : Effects of paternal occupational exposure on spontaneous abortions. Am. J. Pub. Health, 1991, 81 : 1029-1033.

74. LUCIA A., CHICHARRO J.L., PEREZ M., SERRATOSA L., BANDRES F., LEGIDO J.C. : Reproductive function in male endurance athetes / sperm analysis and hormonal profile. J. Appl. Physiol., 1996, 81 : 2627-2636.

75. LUNDSBERG L.S., BRACKEN M.B., BELANGER K. : Occupationally related magnetic field exposure and male subfertility. Am. J. Epidemiol., 1993, 138 : 586. (Abstract).

76. MACLEOD J., WANG Y. : Male fertility potential in terms of semen quality : a review of the past, a study of the present. Fertil. Steril., 1979, 31 : 103-116.

77. MANSON H.J. : Occupational cadmium exposure and testicular endocrine function. Hum. Exp. Toxicol., 1990, 9 : 91-94.

78. McDONALD A.D., McDONALD J.C. ARMSTRONG B., CHERRY N.M., NOLIN A.D., ROBERT D. : Fathers' occupation and pregnancy outcome. Br. J. Ind. Med., 1989, 46 : 329-333.

79. McLACHLAN J.A. : Rodent models for perinatal exposure to diethylstilbestrol and their relation to human disease in the male. In : Herbst A.L., Bern H.A. eds. Developmental Effects of Diethylstilbestrol (DES) in Pregnancy. New York, Thieme-Stratton Inc, $1981:$ 148-157.

80. MENA L., MARIN O., FUENZALIDA S., COTZALS C.C. : Chronic manganese poisoning - clinical picture and manganese turnover. Neurology, 1967, $17: 128-$ 136.

81. MIEUSSET R., GRANDJEAN H., MANSAT A., PONTONNIER F. : Inhibiting effect of artificial cryptorchidism on spermatogenesis. Fertil. Steril., 1985, $43: 574-589$.

82. MIEUSSET R., BUJAN L., MANSAT A., PONTONNIER F., GRANDJEAN H. : Effect of artificial cryptorchidism on sperm morphology. Fertil. Steril., 1987, 47 : 150-155.

83. MIEUSSET R., BUJAN L. : The potential of mild testicular heating as a safe, effective and reversible contraceptive method for men. Int. J. Androl., 1994, 17 :186-191.

84. MIEUSSET R., BUJAN L. : Testicular heating and its possible contributions to male infertility : a review. Int. J. Androl., 1995, 18 : 169-184.

85. MUNKITTRICK K.R., PORT C.B., VAN DER KRAAK G.J., SMITH I.R., ROKOSH D.A. : Impact of bleached kraft mill effluent on population characteristics, liver MFO activity, and serum steroid levels of a Lake Superior white sucker (Catostomus commersoni) population. Can. J. Fish. Aquat. Sci., 1991, 48 : 1371-1380.

86. NELSON C.M.K., BUNGE R.G. : Semen analysis : evidence for changing parameters of male fertility potential. Fertil. Steril., 1974, 25 : 503-507.

87. NEWBOLD R.R., BULLOCK B.C., MCLACHLAN J.A. ; Adenocarcinoma of the rete testis. Diethylstilbestrol-induced lesions of the mouse rete testis. Am. J. Pathol., 1986, 125 : 625-628.

88. NEWBOLD R.R., MCLACHLAN J.A. : Diethylstilbestrol associated defects in murine geniral tract development. In : McLachlan J.A. ed. Estrogens in the Environment II : Influences on Development. New York, Elsevier Science Publisching Co. Inc., 1985 : 288-318.

89. NORDSTROM S., BIRKE E., GUSTAVSSON L. : Reproductive hazards among workers at high voltage substations. Bioelectromagnetics, 1983, 4 : 91-101.

90. OLSEN J., BOLUMAS F., BOLDSEN J., BISANTI L., THE EUROPEAN STUDY GROUP ON INFERTILITY AND SUBFECONDITY. Does moderate alcohol intake reduce fecundability ? A european multicenter study on infertility and subfecondity. Alcohol. Clin. Exp. Res., 1997, 21 : 206-212.

91. OLSEN G.W., BODNER K.M., RAMLOW J.M., ROSS C.E., LIPSHULTZ L.I. : Have sperm counts been reduced 50 percent in 50 years ? A statistical model revisited. Fertil. Steril., 1995, 63 : 887-893. 
92. PAJARINEN J., KARHUNEN P.J., SAVOLAINEN V., LALU K. PENTTILA A., LAIPPALA P. : Moderate alcohol consumption and disorders of hyma spermatogenesis. Alcohol Clin. Exp. Res., 1996, 20 : 332-337.

93. PARAZZINI F., MARCHINI M., LUCHINI L., TOZZI L., MEZZOPANE R., FEDELE L. : Tight underpants and trousers and risk of dyspermia. Int. J. Androl., $1995,18: 137-140$.

94. PETERSON R. : Male reproductive system ontogeny: effects of perinatal exposure to 2, 3, 7,8-TCDD. In : Colborn T., Clement C. eds. Chemically induced alterations in sexual and functionnal development: the Wildlife Human Connection. Princeton Scientific Publishing, 1992 : 1175-1193.

95. POBEL D., VIEL JF. : Case-control study of leukemia among young people near La Hague nuclear reprocessing plant: the environmental hypothesis revisited. Brit. Med. J., 1997, 314 : 101-106.

96. POTASHNIK G., YANI-INBAR I. : Dibromochloropropane (DBCP) : a 8-year reevaluation of testicular function and reproductive performance. Fertil. Steril., 1987, $47: 317-323$.

97. PRASAD M.R., DICZFABUSY N.E. : Gossypol. Int. J. Androl., 1982, 28 (suppl.) : 53-70.

98. RACHOOTIN P., OLSEN J. : The risk of infertility and delayed conception associated with exposures in the Danish workplace. J. Occup. Med, 1983, 25 : 394402.

99. RATCLIFFE J.M., SCHRADER S.M., STEENLAND K., CLAPP D.E., TURNER T. HORNUNG R.W. : Semen quality in papaya workers with long-term exposure to ethylene dibromide. Br. J. Ind. Med., 1987, 44 : 317-326.

100.RODIER J. : Manganese poisoning in Morrocan miners. Br. J. Ind. Med., 1955, 12 : 21.

101.ROWLEY M.J., LEACH D.R., WARNER G.A., HELLER C.G. : Effects of graded doses of ionizing radiation on the human testis. Radiation Research, $1974,59: 665-678$.

102.RUPA D.S., REDDY P.P., REDDI O.S. : Reproductive performance in population exposed to pesticides in cotton fields in India. Environ. Res., 1991, $55: 123$ 128.

103.SANJOSE S., ROMAN E., BERAL V. : Low birthweight and preterm delivery. Scotland, 1981-84 : effect of parents occupation. Lancet, 1991, $338: 428$.

104.SAVITZ D.A., WHELAN E.A., KLECKNER R.C. : Self-reported exposure to pesticides and radiation related to pregnancy outcome - result from National Natality and Fetal Mortality Surveys, Publ. Health Rep., 1989, 104 : 473-477.

105.SCHMID K., LEDERER P., GOËN T, et al. : Internal exposure to hazardous substances of persons from various continents: investigations on exposure to different organochlorine compounds. Int. Arch. Occup. Environ. Health., 1997, $69: 399-406$.
106.SCHUMACHER G.F.B., GILL W.B., HUBBY M.M., BLUOGH R.R. : Semen analysis in males exposed in utero to diethylstilbestrol (DES) or placebo. Reprod. Obstet. Gynecol., 1981, $9:$ 100-101.

107.SHARPE R.M., SKAKKEBAEK N.E. : Are oestrogens involved in falling sperm counts and disorders of the male reproductive tract ? Lancet, 1993, 341 : 1392-1395.

108.SRAM R.J., BINKOVA B., ROSSNER P., RUBES J., TOPINKA J., DEJMEK J. ; Adverse reproductive outcomes from exposure to environmental mutagens. Mutat. Research, 1999, 428 : 203-215.

109.STENCHEVER M.A., WILLIAMSON R.A., LEONARD J. et al. : Possible relationship between in utero diethylstilbestrol exposure and male fertility. Am. J. Obstet. Gynecol., 1981, 140 : 186-193.

110.TAS S., LAUWERYS R., LISON D. : Occupational hasards for the male reproductive system. Crit. Rev. Toxicol., 1996, 26 : 261-307.

111. THONNEAU P., DUCOT B., BUJAN L., MIEUSSET R., SPIRA A. : Heat exposure as a hazard to male fertility. Lancet, 1996, $347:$ 304-305.

112. THONNEAU P., MULTIGNIER L., DUCOT B. et al.: Pesticides exposure and fertility : results of an epidemiological study of French vineyard workers in Alsace. International Worshop: Hormones and endocrine disrupters in food and water : possible effects on human health. Copenhagen, 27-30 may, 2000.

113.TIEMESSEN C.H.T., EVERS J.L.H., BOTS A.S.G.M.: Tight-fitting underwear and sperm quality. Lancet, 1996, 347: 1844-1845.

114. TOPPARI J., LARSEN J.C., CHISTIANSEN P. et al.: Occurrence of abnormalities in the reproductive system of the sons of women exposed to diethylstilbestrol (DES) during pregnancy in male reproductive health and environmental chemicals with estrogenics effects. Danish Environmental Protection Agency, $1995: 37-40$.

115. TORKELSON T.R., SADEK S.E., ROWE V.K. et al. : Toxicologic investigation of 1,2-dibromo-3-chloropropane. Toxicol. Appl. Pharmacol., 1961, 3 : 545-559.

116. VELEZ DE LA CALLE J.F., RACHOU E., LE MARTELOT M.T., DUCOT M., THONNEAU P. Male infertility risk factors in a French military population (results of a population-based casecontrol study). 2000, Soumís.

117. VILLALTA J., BALLESCA J.L., NICOLAS J.M., MARTINEZ DE OSABA M.J. ANTUNEZ E., PIMENTEL C. : Testicular function in asymptomatic chronic alcoholies : relation to ethanol intake. Alcohol Clin. Exp. Res., 1987, 21 : 128-133.

118.VINE M.F. : Worldwide decline in semen quality might be due to smoking. Brit. Med. J., 1996, 312 : 506.

119. VINE M.F., MARGOLIN B.H., MORRISON H.I., HULKA B.S. : Cigarette smoking and sperm density: a meta analysis. Fertil. Steril., 1994, $61: 35-43$. 
120.VINE M.F., TSE C.K.J., HU P.C., TRUONG K.Y. : Cigarette smoking and semen quality. Fertil. Steril., 1996, 65 : 835-842.

121.W.H.O. Congenital Malformations Worlwide : A report from the International Clearinghouse for Birth Defects Monitoring Systems. Oxford, Elsevier, 1991 : 113-118.

122. WHITEHEAD E.D., LEITER E. : Genital abnormalities and abnormal semen analyses in male patients exposed to diethylstilbestrol in utero. J. Urol., 1981, $125: 47-50$.

123.WHORTON M.D., FOLIART D.E. : Mutagenicity, carcinogenicity and reproductive effects of dibromochloropropane (DBCP). Mutat. Res., 1983, 123 : 1330 .

124.WHORTON M.D., KRAUSS R.M., MARSHALL S., MILBY T.H. : Infertility in male pesticide workers. Lancet, $1977,2: 1259$.

125.WILCOX A.J., BAIRD D.D., WEINBERG C.R., HORNSBY P.P., HERBST A.L. : Fertility in men exposed prenatally to diethylstilbestrol. N. Engl. J. Med., 1995, 332 : 1411-1416.

126.WONG O., UTIDJIAN H.M., KARTEN V.S. : Retrospective evaluation of reproductive performance of workers exposed to ethylene dibromide (EDB). J. Oceup. Med., 1979, 21 : 98-102.

127. WYROBEK A.J., WATCHMLARKER G., GORDON L., WONG K., MOORE D., WHORTON D. : Sperm shape abnormalities in carbaryl-exposed employees. Environ. Health Perspect., 1981, 40 : 255-265.

\begin{abstract}
Environment and spermatogenesis
\end{abstract}

L. BUJAN

An alteration of the male reproductive system as well as regional differences in this alterations were recently reported in several studies. This suggests a relationship between environmental conditions and spermatogenesis. Histories of dibromochloropropane distilbene and gossypol are indicative of the influence of occupational and drug exposure, or of the life style on the male reproductive tract. The present review deals with chemical (pesticides, metals,...), physical (ionising and non-ionising radiation's, endogenous heat) and life style factors (exogenous heat, posture, clothing, alcohol, tobacco, stress, sport, nutrition) susceptible to have a deleterious effect on spermatogenesis. Besides, the hypothesis of endocrine disrupters is discussed with inclusion of the information of regional differences from recent studies. While further studies are required to precisely determine the function of the quoted factors in the secular decrease and regional variations of sper. matogenesis, it is suggested that sperm is an indicator of public health and that the principle of precaution is to be considered with the usual implication : 1 ) effective preventive action in occupational world, 2) avoiding of a risk factor discovered during infecondity consulting, 3) non use of substances which innocuity is not proved by toxicologic studies in animals.

Key-words : environment, sperm decline, infertility, sperm, risk factors, occupational risks, life style factors, review 